\title{
Water Properties of Soft Contact Lenses: A Comparative Near-Infrared Study of Two Hydrogel Materials
}

\author{
Jelena Munćan, ${ }^{1}$ Ivana Mileusnić, ${ }^{1}$ Jovana Šakota Rosić, ${ }^{1}$ \\ Aleksandra Vasić-Milovanović, ${ }^{2}$ and Lidija Matija ${ }^{1}$ \\ ${ }^{1}$ Nanolab, Biomedical Engineering Department, Faculty of Mechanical Engineering, University of Belgrade, Kraljice Marije 16, \\ 11120 Belgrade, Serbia \\ ${ }^{2}$ Department for Physics and Electrical Engineering, Faculty of Mechanical Engineering, University of Belgrade, \\ Kraljice Marije 16, 11120 Belgrade, Serbia
}

Correspondence should be addressed to Jelena Munćan; jmuncan@mas.bg.ac.rs

Received 19 February 2016; Accepted 11 May 2016

Academic Editor: Radmila Tomovska

Copyright (C) 2016 Jelena Munćan et al. This is an open access article distributed under the Creative Commons Attribution License, which permits unrestricted use, distribution, and reproduction in any medium, provided the original work is properly cited.

\begin{abstract}
The functionality of soft contact lenses depends strongly on the water content and their water-transport ability. This study was conducted in order to examine the state of water in two sets of soft contact lenses: VSO38, pHEMA Filcon I 1, and VSO50, copolymer of HEMA and VP Filcon II 1 (HEMA = 2-hydroxy-ethyl methacrylate; VP = vinyl pyrrolidone). Hydrogel lenses were studied using near-infrared spectroscopy and the novel Aquaphotomics approach in order to determine the state of water in materials based on their near-infrared spectra. Aquaphotomics approach investigates absorption at specific vibrational bands of water's covalent and hydrogen bonds which can provide information on how the water structure changes with the structural change of the polymer network. Principal component analysis and specific star-chart "aquagram" were used to analyse water spectral pattern in hydrogel materials. The findings show that material VSO38 has water predominantly organized in bound state, while material with higher water content, VSO50, has more free and weakly hydrogen bonded water. Our findings define in detail exact water species existing and interacting with the polymer network. The results show qualitative and quantitative possibilities of Aquaphotomics for better modelling and understanding water behaviour in hydrogel materials.
\end{abstract}

\section{Introduction}

Chemical and physical properties of hydrogels have been the object of extensive research over the past few decades. Hydrogels are commonly used for the manufacture of soft conventional and disposable contact lenses, but they have numerous other applications in biomedicine such as drug delivery systems, artificial implants, wound healing, and sensing applications.

Many of the properties of hydrogels are governed by the interaction between the polymer and the water the polymer absorbs or binds. Specifically, the functionality of soft contact lenses on the human eye depends strongly on the water content and their ability of water-transport and oxygen transmissibility characteristics $[1,2]$.

Currently, following techniques have been used in an attempt to better understand hydration properties of soft contact lenses: differential scanning calorimetry [3-6], differential thermal analysis [4], sorption/desorption experiments [7, 8] nuclear magnetic resonance (NMR) [5, 9-11], and Raman [12] and Fourier transform infrared spectroscopy [13, 14].

The properties of water in hydrogel contact lenses such as the water content, free to bound water ratio, and the extent of dehydration during wear are key determinants of their successful application [2]. However, in spite of extensive research, the state of water in hydrogels still remains poorly understood [10].

The state of water in hydrogels has been described usually in the terms of free and bound water, which is classified further into nonfreezable and freezable part $[2,15]$. The existence and quantification of these different water fractions in the polymer have been determined through the use of differential scanning calorimetry (DSC), thermogravimetric analysis 
(TGA), nuclear magnetic resonance (NMR) spectroscopy, infrared spectroscopy, and chromatography [2].

Generally, at least two different types of water can be present in the hydrogel, which could be described as water strongly interacting with the polymer and water which do not interact at all with the polymer matrix. The bound water exists as tightly and loosely bound, where tightly bound water is associated with water molecules forming hydrogen bonds with the polar groups of polymer or interacting strongly with ionic residues, and as a result it is nonfreezable under normal conditions, whereas loosely bound water is more vaguely defined, covers diverse classes of water species that remain in liquid state below the normal freezing temperature, and usually refers to water molecules in water-swollen polymer systems which are only loosely associated with polar groups through hydrogen bonding but have higher hydrogen bonding energies than that of pure water. Free or bulk water refers to the rest of water molecules, which have hydrogen bonding characteristic of pure water, and is freezable [2].

Tranoudis and Efron outlined the most crucial difference in these types of water from the aspect of application of hydrogels as contact lenses materials: the water which does not participate in transport is water strongly interacting with the polymer [2], which means only loosely bound and free water are available to transport hydrated solutes $[2,3]$. Since the cornea depends solely on the oxygen from atmosphere, biocompatibility of contact lenses and their subsequent application is directly linked to the possible permeation of lens material by oxygen. A hydrogel which is not permeable to water will not be permeable to oxygen either; therefore hydrogel that contains exclusively tightly bound water will not be clinically acceptable because it would not provide adequate oxygen permeability $[3,16]$. In conclusion, free water fraction decides on transport properties of hydrogels $[2,3,16,17]$, and it will affect both diffusion and dehydration behaviour $[6,16]$.

The purpose of present research is to introduce a new, noninvasive, easy-to-use, cost-effective, and rapid method for characterization of soft contact lenses. We here propose the application of near-infrared (NIR) spectroscopy and Aquaphotomics [18] as a novel tool to examine the state of water in hydrogel materials.

Usually, in spectroscopy, water has been considered an obstacle to analytical process of other molecules in aqueous systems $[18,19]$. However, Aquaphotomics has recently emerged as framework to examine the behaviour of water in various aqueous systems through analysis of their NIR spectra.

Aquaphotomics approach is based on near-infrared spectroscopy and multivariate analysis and it determines interactions between water and other components present in various aqueous systems. It has become an effective method for qualitative and quantitative parameters measurements, and its potential lies in accurate description of specific water species characteristic for each material, compound, or solute interacting with water. In Aquaphotomics concept, the spectrum of water serves as molecular mirror of all molecules present in the aqueous system, because the vibrations of water molecules change as a result of vibrations of other molecules interacting with it, and by tracking changes in water molecular vibrations, indirectly information on these other molecules can be extracted as well. The water-light interaction presented in the form of NIR spectra can be used as an enormous source of both physical information and chemical information.

Previous studies have shown that in the range of the first overtone of $\mathrm{OH}$ stretching band a number of characteristic prominent water bands can be observed which are related to specific functions [18]. These water bands, called WAMACs [18], originate from the different water molecular conformations such as free water molecular species, water dimers, trimers, tetramers, or pentamers. Aquaphotomics approach was so far applied for various detection, qualitative descriptions, quantification, dynamic monitoring, and diagnostic purposes [18, 20-26].

However, there is no available publication, yet, which presents the applicability of Aquaphotomics in characterization of hydration properties of hydrogel materials.

Therefore, the objective of this research was to perform a near-infrared spectroscopic characterization using Aquaphotomics approach of the following two classes of hydrogel materials with different nominal water contents (n.w.c.): VSO38, pHEMA Filcon I 1 (n.w.c. 38\%), and VSO50, copolymer of HEMA and VP Filcon II 1 (n.w.c. 48\%) [HEMA = 2hydroxy-ethyl methacrylate; $\mathrm{VP}=$ vinyl pyrrolidone].

The high water content of hydrogel contact lenses makes them ideal to study from Aquaphotomics perspective. It is anticipated that the application of this method could provide insights into water molecular arrangement and lead to more complete understanding of the state of water in hydrogel contact lenses.

\section{Materials and Methods}

2.1. Lenses. All hydrogel contact lens samples were stored in sealed glass vials and equilibrated in phosphate buffered saline (PBS) for at least $24 \mathrm{~h}$ at room temperature $\left(24 \pm 1^{\circ} \mathrm{C}\right)$. PBS is a standard solution used for hydration of commercial contact lenses because it successfully mimics composition and properties of tears.

The used materials belong to one of a series of poly-Hema formulations made by a company, Vista Optics, England. The properties of soft contact lenses materials are given in Table 1.

All lenses were manufactured (lathe-cut) by the same contact lens manufacturer (Optix DOO, Belgrade, Serbia) and had following parameters: BC, 8,60 mm, DIA, 14,20 mm, and $\mathrm{Sph},+1,00 \mathrm{D}$. Six lenses from each of these two materials were used in the experiments.

2.2. Experimental Procedure. Acquisition of near-infrared spectra was done in transmittance mode using minispectrometer Hamamatsu (TG, Cooled NIR-IC9913GC, Hamamatsu, Japan) in the region of $900 \mathrm{~nm}$ to $1700 \mathrm{~nm}$ with the maximum resolution of $7 \mathrm{~nm}$ at integration time of $700 \mu \mathrm{s}$. Transmission fixture TXF-4 (Stellarnet, US) and specially designed holder for contact lenses (Figure 1) were used to ensure fixed central position of lenses during measurements and to prevent environmental influences. 
TABLE 1: Soft contact lens materials used in this research (specifics provided by Vista Optics, England).

\begin{tabular}{lcc}
\hline Material & VSO38 & VSO50 \\
\hline British approved name & pHEMA Filcon I 1 & Copolymer of HEMA and VP Filcon II 1 \\
Water content & $38.1 \% \pm 2$ & $48 \% \pm 2$ \\
Average measured Dk (fatt) & 9 & 15 \\
Expansion ratio radial & $1.163 \pm 0.04$ & $1.270 \pm 0.04$ \\
Expansion ratio diametric & $1.2041 \pm 0.04$ & $1.270 \pm 0.04$ \\
Refractive index wet & $1.4351 \pm 0.001$ & $1.420 \pm 0.001$ \\
Refractive index dry & $1.5178 \pm 0.003$ & $1.524 \pm-0.003$ \\
\hline
\end{tabular}
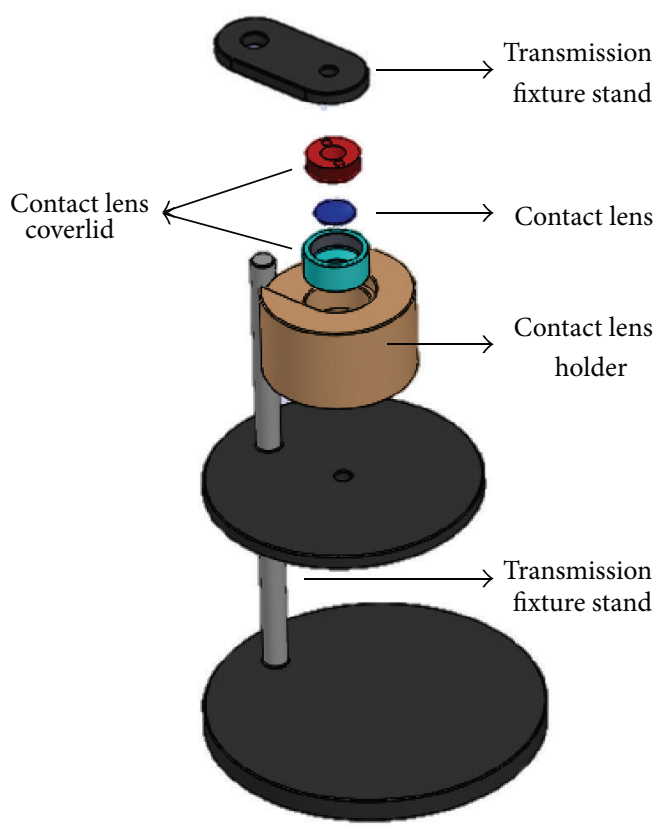

FIGURE 1: Experimental setup for transmittance measurement of soft contact lenses.

Before spectral measurement each lens sample was taken out of its vial and blotted quickly with lint free tissue to remove excessive surface liquid. Experiment was repeated three times, with $24 \mathrm{~h}$ difference in between. The temperature in laboratory was $24 \pm 1^{\circ} \mathrm{C}$.

Five consecutive spectral replicas were acquired for each lens per experiment, giving in total from three experiments 180 spectra ( 3 experimental days $\times 12$ lenses $\times 5$ consecutive replicas). A reference spectrum was recorded before each sample (empty sample holder).

Only the region of the first overtone of water $(1300 \mathrm{~nm}-$ $1600 \mathrm{~nm}$ ) was used in further analysis.

2.3. Data Analysis. Multivariate analysis of absorbance spectra $\left(\log T^{-1}\right)$ was carried out by Pirouette ver. 4.0 (Infometrics, USA) software program.

2.3.1. Spectral Pretreatment. Spectral pretreatment was performed by using Savitzky-Golay smoothing filter [27] using 2nd-order polynomial and 25 points to remove instrumental noise. Mean centering and standard normal variate pretreatment (SNV) [28-30] were applied to dataset before analysis for elimination of scatter effect and suppressing spectral variability due to path length variations in soft contact lenses.

2.3.2. Principal Component Analysis (PCA). Principal component analysis is one of the most commonly used multivariate analysis techniques for revealing internal structure of the data in a way that best explains the variance in the data. Principal components are orthogonal to each other and define a pattern space which explains all the variation in the data [28].

Uncovered principal components (PCs) are presented by scores on 2D scatter plots in a Euclidean plane which allows immediate visualization of where the samples are placed in the PC space and makes the detection of sample groupings or trends easier. Scores plot is discussed together with the corresponding loadings plot. The loadings represent the weight of each of the original variables in determining the direction of each of the PCs or, which is the same as PCs, are defined as the maximum variance directions, which of the original variables vary the most for the samples with different score values on each of the components [31].

The PCA analysis was performed on pretreated spectral data and validation was performed by leave-three-out cross validation.

2.3.3. Aquagrams. The absorbance values at specific water matrix coordinates (WAMACs) define the water spectral pattern (WASP) [18]. The visualization of WASP can be done by star chart called aquagram [32].

Aquagrams were calculated in order to show the differences of the absorbance values at the water matrix coordinates for the classes of two different hydrogel materials. The aquagram displays averaged normalized absorbance values for different sample classes at selected water bands on the axes originating from the center of the graph.

Normalized absorbance was calculated using the following equation:

$$
A_{\lambda^{\prime}}=\frac{A_{\lambda}-\mu_{\lambda}}{\sigma_{\lambda}}
$$

where $A_{\lambda}, \mu_{\lambda}$, and $\sigma_{\lambda}$ are absorbances, mean, and standard deviation of all pretreated spectra, respectively, at a given wavelength.

Aquagrams were prepared in Microsoft Office Excel 2013 (Microsoft C., Redmond, WA, USA). 


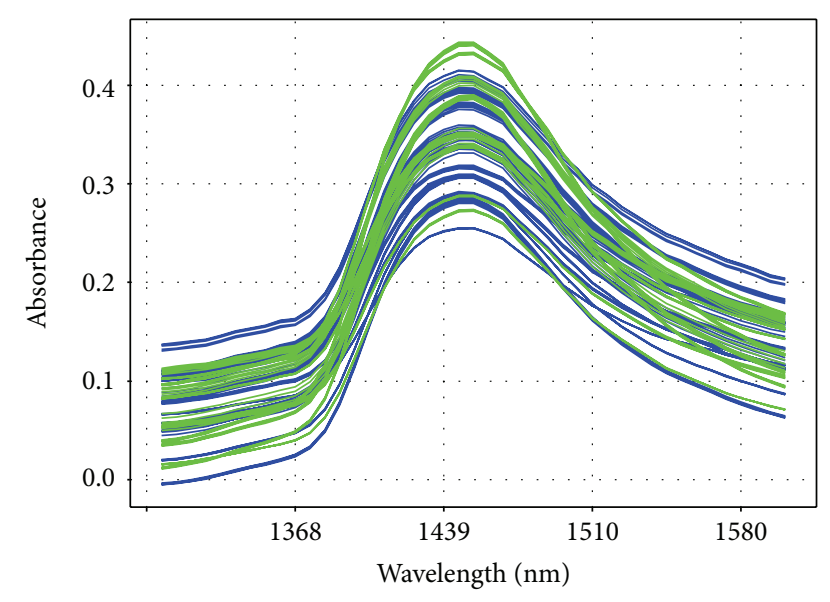

Figure 2: Raw spectra of contact lenses in the region of 1300$1600 \mathrm{~nm}$. Spectra of VSO38 contact lenses are presented with blue lines, while those of VSO50 are presented with green lines.

\section{Results and Discussion}

In this study, we applied NIR spectral analysis and Aquaphotomics to enquire about differences between two types of contact lenses made of hydrogel materials, VSO38, pHEMA Filcon I 1, and VSO50, copolymer of HEMA and VP Filcon II 1.

Using specially designed experimental setup NIRS was then used noninvasively to record spectra of contact lenses. All lenses' spectra were acquired at room temperature over the course of three days, in hydrated state, after equilibration for $24 \mathrm{~h}$ at least in PBS solution to mimic physiological conditions.

Aquaphotomics approach utilizes usually only the region of the first overtone of water ranging from $1300 \mathrm{~nm}$ to $1600 \mathrm{~nm}$. We concentrated our study on this water absorbance region where more details are unraveled for water species assignment [18].

The raw absorbance $\log (1 / T)$ spectra of the contact lenses are plotted in Figure 2. The strong absorption near $1450 \mathrm{~nm}$ is assigned to the combination of antisymmetric and symmetric stretching modes of water, and this region is generally referred to as the first overtone of the $\mathrm{OH}$ stretch $[33,34]$. Thus, all lenses' spectra are dominated by the water absorption.

The differences in the spectra of the lenses are caused by both physical and chemical properties of water in polymer. In the raw spectra (Figure 2) significant scatter effect is evident (baseline offset) which can be caused by the path length changes due to the uncontrollable physical variations such as variation in thickness of the lens due to the different swelling rate or due to the sample morphology (surface roughness/shape). Such variations are unrelated to the chemical response, but it should be noted that, due to the impact of the physical properties on the spectral response, the NIRS can be used to measure changes in such attributes as well.

Standard normal variate transformation is then used on spectral dataset to correct the influence of scatter and the

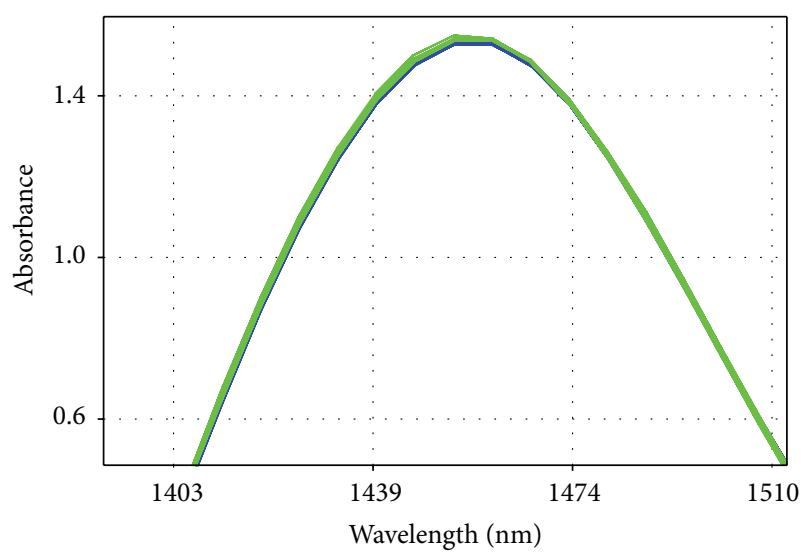

FIgURE 3: Absorbance spectra of hydrated contact lenses in the wavelength region of $1300-1600 \mathrm{~nm}$ after mean centering and standard normal variate transformation. Spectra of VSO38 contact lenses are presented with blue lines, while those of VSO50 are presented with green lines.

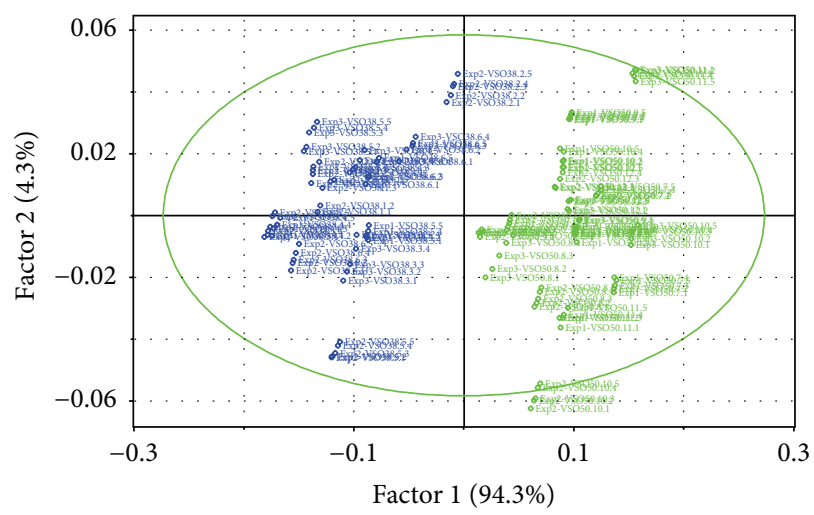

FIGURe 4: Principal component analysis of hydrogel contact lenses. Spectral data presented on scores plot of PC1 factor and PC2 factor show the classification power of this technique. The plot shows good separation between the classes of VSO38 contact lenses (blue scores) and VSO58 contact lenses (green scores).

spectra of contact lenses in the region of first overtone (1300$1600 \mathrm{~nm}$ ) after mean centering and standard normal variate transformation are shown in Figure 3.

To present numerical explanation of the difference between the hydrogel materials VSO38 pHEMA Filcon I 1 and VSO50 copolymer of HEMA and VP Filcon II 1, spectra of lenses were analysed using principal component analysis. The first two principal components described more than $98.6 \%$ of the variation.

The results of PCA analysis presented in Figures 4 and 5 show scores and corresponding loadings plot.

Here it can be seen from the scores plot that principal component analysis of the NIR spectral data classified all samples of the same contact lenses into two distinct groups: all VSO38 scores lenses are located in the negative part of $\mathrm{PC} 1$ factor, while spectral scores of VSO50 lenses are in the positive part. 


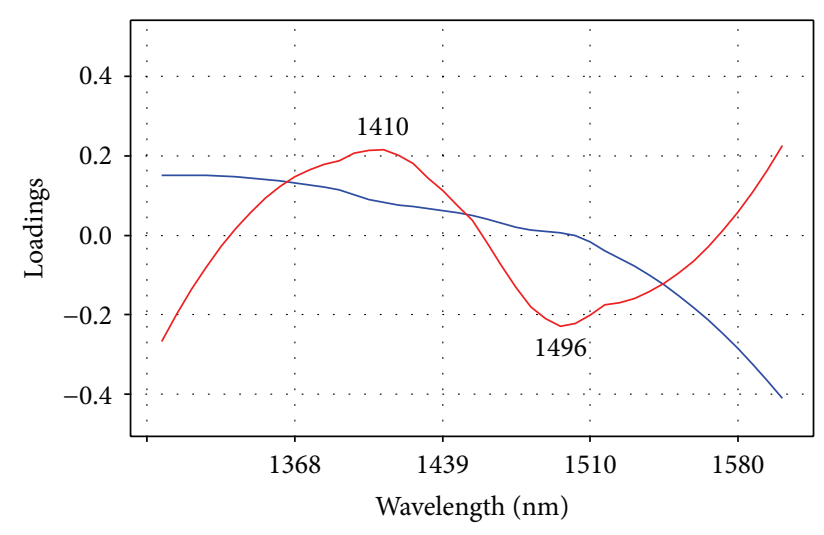

FIGURE 5: Principal component (PC) loadings plotted as a function of wavelength. This plot shows the importance of certain wavelengths in the respective spectral pattern of each PC. First two PC factors described $98,6 \%$ of the variations in the dataset with PC1 factor (blue line) describing 94,3\% and PC2 factor (red line) describing $4.3 \%$.

The scores plot illustrates that PC1, which explains 94.3\% of total variance and is related to water, distinguishes the two hydrogel materials. This loading vector presents water spectral changes related to the state of water in the lenses and it is highly dependent on properties of materials.

For PCl, no characteristic peaks were observed, it shows steady decline with the longer wavelengths, and crosses zero point at approximately $1500 \mathrm{~nm}$. The spectral pattern of PC1 loading has negative scores for VSO38 pHEMA Filcon 1 contact lenses, which corresponds to higher absorbances in the wavelength range between $1491 \mathrm{~nm}$ and $1510 \mathrm{~nm}$.

These wavelengths are introduced as strongly hydrogen bonded water $[35,36]$. The positive scores of VSO50 copolymer of HEMA and VP Filcon II 1 in the spectral pattern of PC1 suggest more free water.

These findings are consistent with the existing data on low and medium water content soft contact lenses; that is, increasing water content will result in an increase in only free water content [2]. These results will be more discussed later.

On the scores plot from Figure 4, another trend can be observed. Along direction of PC2 it is evident that all lenses have higher scores for consecutive replicas in the experiment. This suggests that PC2 loading vector presents water spectral changes caused by the influence of irradiation. Even though the irradiation time was very short (700 ms per one replica), the lenses show fast dehydration during measurements. The main features of PC2 loading are positive absorbance at $1410 \mathrm{~nm}$ and negative absorbance at $1496 \mathrm{~nm}$. According to Segtnan et al. the two major spectral changes of water around $1412 \mathrm{~nm}$ and $1491 \mathrm{~nm}$ are a function of temperature [35] and, in this study, irradiation. These changes occur in phase with each other in opposite directions, where the two states represent weaker and stronger hydrogen bonding at $1412 \mathrm{~nm}$ and $1491 \mathrm{~nm}$, respectively, and these two species are perfectly correlated; that is, the concentration of one species increases at the expense of the other, when the temperature is changed. Due to the low resolution of the instrument $(7 \mathrm{~nm})$, in our experiment, this spectral feature occurs at $1410 \mathrm{~nm}$.
In order to compare how dehydration process occurs in different materials during measurements, the standard deviation was calculated for the presented PC2 scores of each class of samples. The results showed slightly higher standard deviation for VSO50 material $(\mathrm{SD}=0.025)$ compared to VSO38 material $(\mathrm{SD}=0.021)$, indicating probably faster changes in the water state of VSO50 material, which could be due to higher content of unbound water that can more easily be extracted or escape as vapour, but more temperature and time controlled studies would be needed to assert this.

To investigate detailed characteristics of water organization in the contact lenses, the obtained NIR spectra were subjected to analysis of the absorbance pattern at specific water bands, named water matrix coordinates (WAMACs). Tsenkova has defined 12 characteristic water wavelength ranges which cover various conformations of water molecules and are very useful to present water spectral pattern [18]. The ranges of WAMACs were defined as follows: $\mathrm{Cl}$ (1336-1348 nm: v3, $\mathrm{H}_{2} \mathrm{O}$ asymmetric stretching vibration), C2 (1360-1366 nm: water solvation shell, $\mathrm{OH}-$ $\left.\left(\mathrm{H}_{2} \mathrm{O}\right)_{1,2,4}\right)$, C3 (1370-1376 nm: $\nu 1+\nu 3$, symmetrical stretching fundamental vibration and $\mathrm{H}_{2} \mathrm{O}$ asymmetric stretching vibration), C4 (1380-1388 nm: water solvation shell, $\mathrm{OH}-$ $\left(\mathrm{H}_{2} \mathrm{O}\right)_{1,4}$ and superoxide, $\left.\mathrm{O}_{2}-\left(\mathrm{H}_{2} \mathrm{O}\right)_{4}\right), \mathrm{C} 5(1398-1418 \mathrm{~nm}$ : S0, free water and free $\mathrm{OH}-), \mathrm{C} 6$ (1421-1430 nm: $\mathrm{H}-\mathrm{OH}$ bend and O … O), C7 (1432-1444 nm: S1), C8 (1448-1454 nm: water solvation shell, $\left.\mathrm{OH}-\left(\mathrm{H}_{2} \mathrm{O}\right)_{4,5}\right)$, C9 (1458-1468 nm: S2), C10 (1472-1482 nm: S3), C11 (1482-1495 nm: S4), and C12 (1506$1516 \mathrm{~nm}: \nu 1, \nu 2$, symmetrical stretching fundamental vibration, and doubly degenerate bending fundamental), where $S i$ describes number of hydrogen-bonded molecules $[18,26]$.

Figure 6 shows the aquagrams of the hydrogel contact lenses representing the average of the pretreated spectra of the individual lenses across all replicas and experiments, at selected 12 characteristic water wavelengths from the WAMACs regions.

The aquagram of VSO38 contact lenses differs mainly in absorbances at longer wavelengths at C10, $1474 \mathrm{~nm}, \mathrm{Cl1}$, $1488 \mathrm{~nm}$, and C12, $1510 \mathrm{~nm}$, suggesting differences in the water structures like S3 and S4 (water molecules with three and four hydrogen bonds, resp.) and strongly bonded water. The aquagram of VSO50 contact lenses shows mainly higher absorbances at shorter wavelengths which means these lenses contain more free water molecules, more free $\mathrm{OH}$, and more weakly hydrogen bonded water involved in ion hydration and water solvation structures $[18,36]$.

In the context of existing literature on the state of water in low and medium water content hydrogel lenses, our new results provided with near-infrared spectroscopy and Aquaphotomics confirm that the materials vary in the states of bound and free water, where in material with higher water content more free water exists in addition to tightly bound water, which already occupies the available sites in the polymer.

The resulting state of water in VSO38 pHEMA Filcon 1 material is due to the hydroxyl groups $\left(-\mathrm{CH}_{2} \mathrm{CH}_{2} \mathrm{OH}\right)$ of pHEMA. At this location hydrogen bonding with water molecules occurs, causing them to be drawn into the polymer matrix [37]. According to Lee et al. most of water in pHEMA 




FIgURE 6: Aquagrams of the contact lenses calculated at selected wavelengths. The averaged spectra of lenses across all experiments show differences in water spectral pattern for different hydrogel materials (VSO38 contact lenses, VSO38.1 to VSO38.6, and VSO50 contact lenses, VSO50.7 to VSO50.12).

gels with low water content exists as bound water [38], which is in agreement with our results. Vinyl pyrrolidone is one of the monomers commonly used to increase water content in soft contact lenses materials based on pHEMA, but increasing water content increases only free water fraction [2]. In VSO50, copolymer of HEMA and VP Filcon II 1, the higher water content is achieved due to the presence of amide moiety (pyrrolidone group - $\mathrm{NCOCH}_{2}$-), which is very polar and two molecules of water can become hydrogen-bonded to it [37]. However, amide group does not bind water as strongly as hydroxyl group and that is why VSO50 contact lenses contain more loosely bound and free water when compared to VSO38.

When considering the physiological response to contact lenses, the water content is the most important property defining their clinical behaviour. As we already mentioned the solubility of compounds, mostly important oxygen, is a function of the content of free water in a hydrogel. The larger amount of loosely bound and free water seen in the aquagrams of VSO50 as compared to VSO38 suggests more water available for oxygen transport. However, the differences observed in dehydration rate (PC2 scores, Figure 2) indicate faster changes in VSO50 material, again probably due to the less strong binding of water molecules to amide group. These results are in agreement with the findings of faster translational motions in hydrogels with higher water content [11] and higher evaporation rates may be seen as a problem for lens stability and comfort [37, 39].

Since such state of water is useful from one point of view and from another point of view it can lead to interaction with various solutes in tears leading to depositions of proteins and lipids on the contact lens surface, it is difficult to predict exact behaviour of both types of material in general based solely on the state of water. It is rather a complex interaction of various factors and additional clinical work will be undertaken to explore this matter further.

Our most important findings are that the differences in water states and water/polymer interaction in hydrogels can be analysed using near-infrared spectroscopy and Aquaphotomics. Our contribution defines in more detail exact water species existing and interacting with the polymer network, which Aquaphotomics approach provides. The limitation of this study is that the measurements are done on hydrogels swollen in PBS solution, which can contribute to the higher levels of tightly bound water as it was shown for HEMA lenses swollen in aqueous sodium chloride solution compared to swelling in distilled water [38]. However, to mimic the physiological conditions better, we have chosen to use PBS. The other limitation is the instrument accuracy, because it provides real-time, fast acquisition at the expense of resolution. And finally this study is limited to only two hydrogel materials.

Further accumulation of experimental data on water behaviour in hydrogels will provide valuable insights into detailed picture of water organization and, furthermore, into the hydrogel structures providing optimal functionality of hydrogel contact lens materials.

\section{Conclusions}

In this study, we showed that NIR spectroscopy and Aquaphotomics approach could be used successfully for finding the differences in water state of contact lenses made of different hydrogel materials based on their NIR spectra. The main findings are defined dominant water species in hydrogel lenses of low and medium water content.

We presented here new approach for studies of hydration properties of hydrogel based contact lenses where nearinfrared spectra allow monitoring of water species interacting with polymer matrix.

This approach can be used as a common platform for comparison of hydrogel materials in general in a nondestructive, real-time, and cost-effective manner. In addition it could also be used for dynamic studies of hydrogels during swelling and dehydration, as well as in quantitative parameters measurements.

\section{Competing Interests}

The authors declare that there are no competing interests regarding the publication of this paper.

\section{Acknowledgments}

The authors gratefully acknowledge Ministry of Science and Education of Republic of Serbia for financially supporting this research through Project no. III45009. The authors thank Dr. Dragomir Stamenković and Optix DOO, Belgrade, Serbia, for fabrication of soft contact lenses. 


\section{References}

[1] G. Andrasko, "Hydrogel dehydration in various environments," International Contact Lens Clinic, vol. 10, no. 6, pp. 22-28, 1983.

[2] I. Tranoudis and N. Efron, "Water properties of soft contact lens materials," Contact Lens and Anterior Eye, vol. 27, no. 4, pp. 193208, 2004.

[3] D. Mirejovsky, A. S. Patel, and G. Young, "Water properties of hydrogel contact lens materials: a possible predictive model for corneal desiccation staining," Biomaterials, vol. 14, no. 14, pp. 1080-1088, 1993.

[4] W. Roorda, "Do hydrogels contain different classes of water?" Journal of Biomaterials Science. Polymer edition, vol. 5, no. 5, pp. 383-395, 1994.

[5] A. Woźniak-Braszak, M. Kaźmierczak, M. Baranowski, K. Hołderna-Natkaniec, and K. Jurga, "The aging process of hydrogel contact lenses studied by ${ }^{1} \mathrm{H}$ NMR and DSC methods," European Polymer Journal, vol. 76, pp. 135-146, 2016.

[6] K. Krysztofiak and A. Szyczewski, "Study of dehydration and water states in new and worn soft contact lens materials," Optica Applicata, vol. 44, no. 2, pp. 237-250, 2014.

[7] Y.-M. Sun and H.-L. Lee, "Sorption/desorption properties of water vapour in poly(2-hydroxyethyl methacrylate): 1 . Experimental and preliminary analysis," Polymer, vol. 37, no. 17, pp. 3915-3919, 1996.

[8] Y.-M. Sun, "Sorption/desorption properties of water vapour in poly(2-hydroxyethyl methacrylate): 2 . Two-stage sorption models," Polymer, vol. 37, no. 17, pp. 3921-3928, 1996.

[9] D. W. Larsen, J. W. Huff, and B. A. Holden, "Proton NMR relaxation in hydrogel contact lenses: correlation with in vivo Lens dehydration data," Current Eye Research, vol. 9, no. 7, pp. 697-706, 1990.

[10] P. McConville and J. M. Pope, " $1 \mathrm{H}$ NMR T 2 relaxation in contact lens hydrogels as a probe of water mobility," Polymer, vol. 42, no. 8, pp. 3559-3568, 2001.

[11] R. Barbieri, M. Quaglia, M. Delfini, and E. Brosio, "Investigation of water dynamic behaviour in poly(HEMA) and poly(HEMAco-DHPMA) hydrogels by proton T2 relaxation time and selfdiffusion coefficient n.m.r. measurements," Polymer, vol. 39, no. 5, pp. 1059-1066, 1998.

[12] Y. Sekine and T. Ikeda-Fukazawa, "Structural changes of water in a hydrogel during dehydration," The Journal of Chemical Physics, vol. 130, no. 3, Article ID 034501, 2009.

[13] L. Yin, Z. Zhao, Y. Hu et al., "Polymer-protein interaction, water retention, and biocompatibility of a stimuli-sensitive superporous hydrogel containing interpenetrating polymer networks," Journal of Applied Polymer Science, vol. 108, no. 2, pp. 1238-1248, 2008.

[14] S. Anbudayanidhi, S. K. Nayak, and S. Mohanty, "Synthesis and water state characterization of polysodium acrylate/cellulose microfibril hydrogels," Journal of Thermoplastic Composite Materials, vol. 27, no. 5, pp. 573-585, 2014.

[15] D. G. Pedley and B. J. Tighe, "Water binding properties of hydrogel polymers for reverse osmosis and related applications," British Polymer Journal, vol. 11, no. 3, pp. 130-136, 1979.

[16] C. Manetti, L. Casciani, and N. Pescosolido, "Diffusive contribution to permeation of hydrogel contact lenses: theoretical model and experimental evaluation by nuclear magnetic resonance techniques," Polymer, vol. 43, no. 1, pp. 87-92, 2002.

[17] D. Mirejovsky, A. S. Patel, and D. D. Rodriguez, "Effect of proteins on water and transport properties of various hydrogel contact Lens materials," Current Eye Research, vol. 10, no. 3, pp. 187-196, 1991.

[18] R. Tsenkova, "Introduction: aquaphotomics: dynamic spectroscopy of aqueous and biological systems describes peculiarities of water," Journal of Near Infrared Spectroscopy, vol. 17, no. 6, pp. 303-313, 2009.

[19] H. Büning-Pfaue, "Analysis of water in food by near infrared spectroscopy," Food Chemistry, vol. 82, no. 1, pp. 107-115, 2003.

[20] B. Jinendra, K. Tamaki, S. Kuroki, M. Vassileva, S. Yoshida, and R. Tsenkova, "Near infrared spectroscopy and aquaphotomics: novel approach for rapid in vivo diagnosis of virus infected soybean," Biochemical and Biophysical Research Communications, vol. 397, no. 4, pp. 685-690, 2010.

[21] C. Esquerre, A. A. Gowen, C. P. O'donnell, and G. Downey, "Initial studies on the quantitation of bruise damage and freshness in mushrooms using visible-near-infrared spectroscopy," Journal of Agricultural and Food Chemistry, vol. 57, no. 5, pp. 1903-1907, 2009.

[22] A. Slavchev, Z. Kovacs, H. Koshiba et al., "Monitoring of water spectral pattern reveals differences in probiotics growth when used for rapid bacteria selection," PLoS ONE, vol. 10, no. 7, Article ID e0130698, 2015.

[23] R. Tsenkova, "AquaPhotomics: water absorbance pattern as a biological marker for disease diagnosis and disease understanding," NIR News, vol. 18, no. 2, pp. 14-16, 2007.

[24] R. Tsenkova, "AquaPhotomics: water absorbance pattern as a biological marker," NIR News, vol. 17, no. 7, p. 13, 2006.

[25] E. Chatani, Y. Tsuchisaka, Y. Masuda, and R. Tsenkova, "Water molecular system dynamics associated with amyloidogenic nucleation as revealed by real time near infrared spectroscopy and aquaphotomics," PLoS ONE, vol. 9, no. 7, Article ID e101997, 2014.

[26] N. Goto, G. Bazar, Z. Kovacs et al., "Detection of UV-induced cyclobutane pyrimidine dimers by near-infrared spectroscopy and aquaphotomics," Scientific Reports, vol. 5, article 11808, 2015.

[27] A. Savitzky and M. J. E. Golay, "Smoothing and differentiation of data by simplified least squares procedures," Analytical Chemistry, vol. 36, no. 8, pp. 1627-1639, 1964.

[28] H. Martens and M. Martens, Multivariate Analysis of Quality: An Introduction, John Wiley \& Sons, New York, NY, USA, 2001.

[29] E. Li-Chan, J. Chalmers, and P. Griffiths, Applications of Vibrational Spectroscopy in Food Science, John Wiley \& Sons, New York, NY, USA, 2011.

[30] R. J. Barnes, M. S. Dhanoa, and S. J. Lister, "Standard normal variate transformation and de-trending of near-infrared diffuse reflectance spectra," Applied Spectroscopy, vol. 43, no. 5, pp. 772$777,1989$.

[31] F. Marini, Ed., Chemometrics in Food Chemistry, vol. 28, Newnes, 2013.

[32] R. Tsenkova, "Aquaphotomics: water in the biological and aqueous world scrutinised with invisible light," Spectroscopy Europe, vol. 22, no. 6, p. 6, 2010.

[33] B. G. Osborne, T. Fearn, and P. H. Hindle, Practical NIR Spectroscopy with Applications in Food and Beverage Analysis, Longman Scientific and Technical, New York, NY, USA, 1993.

[34] J. Workman Jr. and L. Weyer, Practical Guide to Interpretive Near-Infrared Spectroscopy, CRC Press, 2007.

[35] V. H. Segtnan, Š. Šašić, T. Isaksson, and Y. Ozaki, "Studies on the structure of water using two-dimensional near-infrared correlation spectroscopy and principal component analysis," Analytical Chemistry, vol. 73, no. 13, pp. 3153-3161, 2001. 
[36] A. A. Gowen, R. Tsenkova, C. Esquerre, G. Downey, and C. P. O'Donnell, "Use of near infrared hyperspectral imaging to identify water matrix co-ordinates in mushrooms (Agaricus bisporus) subjected to mechanical vibration," Journal of Near Infrared Spectroscopy, vol. 17, no. 6, pp. 363-371, 2009.

[37] C. Maldonado-Codina and N. Efron, "Hydrogel lenses-material and manufacture: a review ," Optometry in Practice, vol. 4, pp. 101-115, 2003.

[38] H. B. Lee, M. S. Jhon, and J. D. Andrade, "Nature of water in synthetic hydrogels. I. Dilatometry, specific conductivity, and differential scanning calorimetry of polyhydroxyethyl methacrylate," Journal of Colloid and Interface Science, vol. 51, no. 2, pp. 225-231, 1975.

[39] S. Kanome, "Fundamental chemistry and physical properties of polymer material," in Menicon Toyo's 30th Anniversary Compilation of Research Reports, S. Iwata, Ed., pp. 109-138, MeniconToyo Contact Lens Co. Ltd., Nagoya, Japan, 1982. 



\section{Hindawi}

Submit your manuscripts at

http://www.hindawi.com

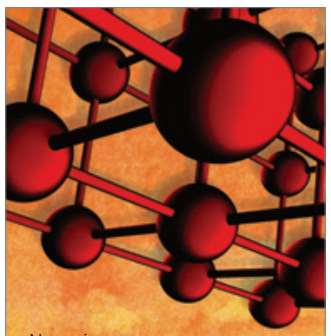

Materials Science and Engineering
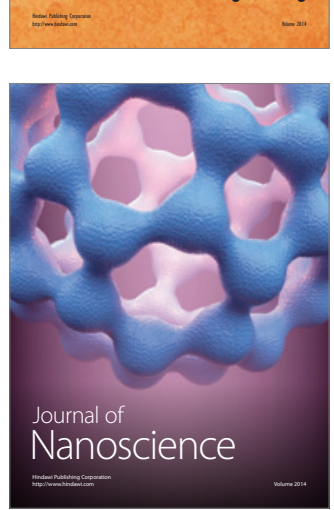
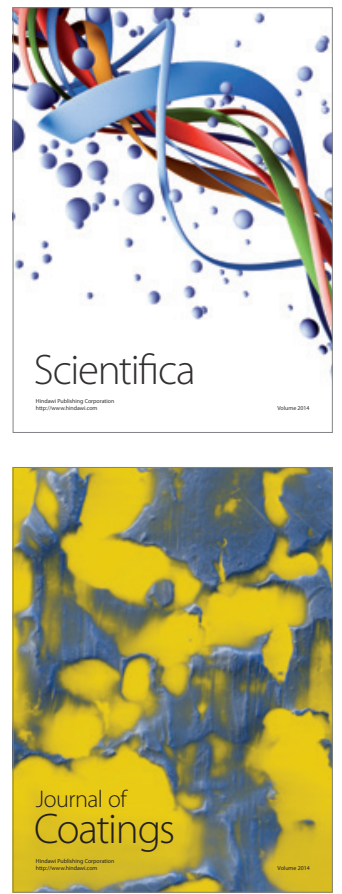


The Scientific World Journal
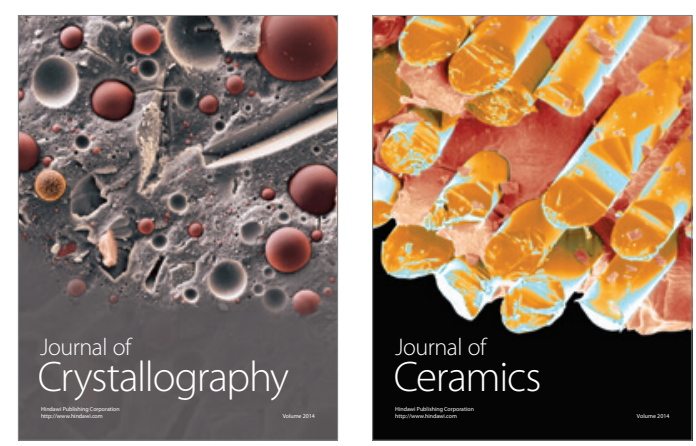
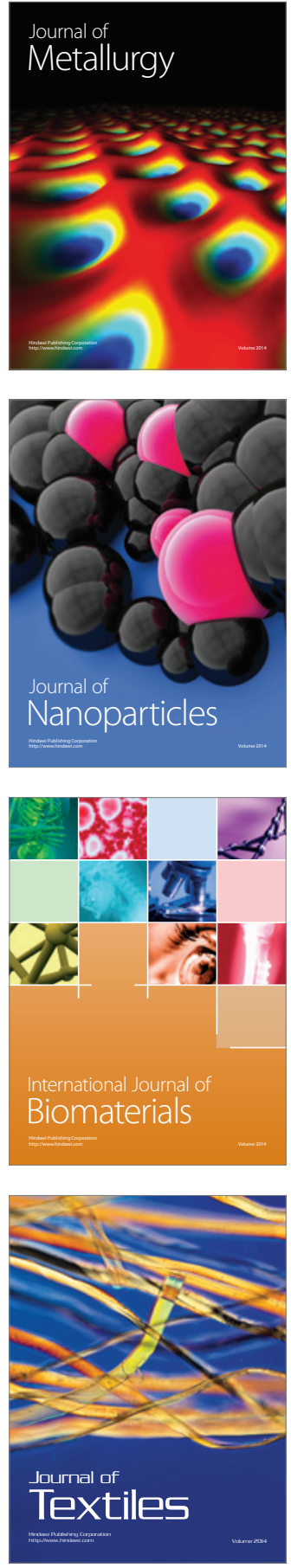